\title{
MYXOMYCETES DA CHAPADA DO ARARIPE (CRATO - CE, BRASIL)
}

\author{
Laise de Holanda Cavalcanti ${ }^{1}$ \\ Jair Putzke ${ }^{2}$
}

\section{Recebido em 07/07/1998. Aceito em 25/11/1998}

\begin{abstract}
RESUMO - (Myxomycetes da Chapada do Araripe (Crato, CE. Brasil)). Foi efetuado um levantamento dos Myxomycetes ocorrentes em área de floresta no município do Crato, CE ( $7^{\circ} 13^{\prime} 53^{\prime \prime}$ S; $39^{\circ} 24^{\prime} 28^{\prime \prime} \mathrm{W}$; Alt. $\left.422 \mathrm{~m}\right)$, assinalando-se as seguintes famílias e gêneros: Ceratiomyxaceae (Ceratiomyxa. I sp.); Cribrariaceae (Cribraria.1sp.. Dictrdium. 1sp.); Didymiaceae (Didymium, 2sp.); Enteridiaceae (Dictydiaethalium, 1sp.); Physaraceae (Badhamia, 2sp., Fuligo, 2sp., Physarum, 5sp.); Stemonitaceae (Comatricha, 3sp., Stemonitis, 6sp.); Trichiaceac (Arcyria, 3sp., Hemitrichia, 2sp., Perichaena, Isp.). Constatou-se preferência das espécies para frutificar em troncos mortos de dicotiledôneas, seguindo-se restos de palmeiras e folhedo. O levantamento eleva para 30 o número de espécies referidas para o Ceará. Uma família e 19 espécies são novos registros para o Estado e Arcyria magna var. rosea Rex é nova referência para o Brasil. São apresentados comentários, chave de identificação e distribuição geográfica das espécies no Nordeste do Brasil.
\end{abstract}

Palavras - chave - Myxomycetes, floresta tropical, corologia

\begin{abstract}
Myxomycetes from Chapada do Araripe (Crato, CE . Brazil)). A survey on Myxomycetes was made in the woods of Crato Municipality. State of Ceará, Brazil ( $7^{\circ} 13^{\prime} 53^{\prime \prime} \mathrm{S}$; $39^{\circ} 24^{\prime} 28^{\prime \prime}$ W; Alt. $422 \mathrm{~m}$ ), when the following families and genera were registered: Ceratiomyxaceae (Ceratiomyxa. 1sp.); Cribrariaceae (Cribraria, Isp.. Dictydium, Isp.); Didymiaceae (Didymium, 2sp.); Enteridiaceae Dictydiaethalium, 1sp.); Physaraceae (Badhamia. 2sp., Fuligo, 2sp., Physarum, 5sp.); Stemonitaceae Comatricha, 3sp.., Stemonitis, 6sp.); Trichiaceae (Arcyria, 3sp., Hemitrichia, 2sp., Perichaena, Isp.).The species sporulated mostly on dead dicotyledones wood, followed by monocotyledones (Arecaceae) debris and litter. The survey raises the number of registers referred to the State of Ceará to 30 species. One family and 19 species are new records for that State while Arcyria magna var, rosea Rex represents a new record for Brazil. Key for the species, comments and geographical distribution in Northeast Brazil are presented.
\end{abstract}

Key words - Myxomycetes, tropical forest, chorology

\section{Introdução}

Os estudos efetuados até o momento sobre os Myxomycetes do Nordeste do Brasil relatam a ocorrência de sete espécies para Alagoas, 60 para a Bahia, 20 para o Ceará,

Universidade Federal de Pernambuco, UFPE, CCB. Departamento de Botânica, Cidade Universitária, CEP 50670 - 901, Recife. PE. Brasil. e-mail: Ihc @ netpe.com.br (CNPq processo 300626 / 79-7)

Universidade de Santa Cruz do Sul, UNISC, Av. Independência 2293. C. Postal 188, CEP 96815-900, Santa Cruz do Sul, RS, Brasil 
três para o Maranhão, 37 para a Paraíba , 145 para Pernambuco e nove para o Rio Grande do Norte (Cavalcanti 1996 ).

Recentemente, Alves \& Cavalcanti (1996) forneceram os primeiros registros da presença de myxomycetes no Estado do Ceará, baseados em coletas realizadas no município do Crato, Microrregião do Cariri. As autoras deram atenção às espécies que utilizam palmeiras como substrato e referem cinco famílias, nove gêneros e 12 espécies que frutificam sobre Acrocomia intumescens Drude, Copernicia prunifera (Mill.) H. E. Moore, Mauritia flexuosa L.f. e Orbygnia phalerata Mart. No mesmo ano, Cavalcanti (1996) informa a ocorrência de seis famílias e 11 gêneros , aos quais pertencem 20 espécies assinaladas para o mesmo Estado, sem contudo nomeá-las.

Como parte dos estudos sobre a mixomicota cearense, foi realizado no município do Crato um levantamento das espécies lignícolas e folíícolas de diferentes famílias de angiospermas. Os resultados são apresentados no presente trabalho, elevando para $30 \mathrm{o}$ número de espécies de Myxomycetes de ocorrência conhecida para o Estado do Ceará.

\section{Material e métodos}

O município do Crato ( $7^{\circ} 13^{\prime} 53^{\prime \prime}$ S; $39^{\circ} 24^{\prime} 28^{\prime \prime}$ W) localiza-se ao sul do Estado do Ceará, na zona fisiográfica do Cariri. A sede municipal situa-se a $422 \mathrm{~m}$ de altitude e a topografia do município apresenta-se acidentada em cerca de $2 / 3$, sendo o restante constituído por vales e tabuleiros (FIBGE 1977).

A microrregião do Cariri apresenta cobertura vegetal dos tipos floresta (perenifólia, caducifólia e subcaducifólia), caatinga (hiperxerófila e hipoxerófila), cerrado e cerradão, sendo o principal acidente geográfico a Chapada do Araripe, segmento da Serra do Ibiapaba. A vegetação estudada é uma floresta tropical perenifólia que se desenvolve sob clima de temperaturas amenas, com precipitações abundantes e umidade atmosférica elevada (SUDEC 1972).

As coletas foram realizadas ao final da estação chuvosa, em três localidades do município conhecidas como Sítio Fundão, Sítio Luanda e Guaribas. Além de se pesquisar a presença de frutificações em troncos mortos, caídos ou em pé, folhedo e casca de árvores vivas, foram lançados ao acaso 12 quadrados de $1 \mathrm{~m}^{2}$ de área, recolhendo-se o folhedo por eles delimitados, o qual foi acondicionado em sacos plásticos, determinandose posteriormente a biomassa e o teor de umidade. Seguiu-se a metodologia indicada por Cavalcanti (1974) e Farr (1976) para a coleta, acondicionamento, herborização e análise das amostras. Para identificação das espécies, utilizou-se Lister (1925), Martin \& Alexopoulos (1969), Farr (1976) e Yamamoto \& Nannenga-Bremekamp (1995), seguindo-se a proposta de classificação de Martin et al. (1983).

A distribuição geográfica das espécies no Nordeste do Brasil baseou-se nos trabalhos de Torrend (1915; 1916), Farr (1960), Gottsberger (1968), Cavalcanti (1976), Pôrto (1982), Cavalcanti \& Araújo (1985), Cavalcanti \& Marinho (1985), Cavalcanti \& Oliveira (1985), Santos et al. (1986), Cavalcanti \& Brito Júnior (1990), Góes Neto (1994), Alves \& Cavalcanti (1996) e Cavalcanti (1996).

\section{Resultados e discussão}

No presente estudo, realizado ao final da estação chuvosa, em apenas quatro dias foram coletadas 30 espécies de Myxomycetes, distribuídas em 13 gêneros, de sete 
diferentes famílias, contradizendo as informações de Alexopoulos (1970) sobre a baixa diversidade de espécies em florestas tropicais. Além de evidenciar rica mixomicota para a área estudada, o número de espécies equivale ao encontrado em levantamentos efetuados em vegetação de cerrado e floresta nas Regiões Sudeste e Sul do Brasil, em períodos muito mais longos (12 meses), que abrangeram diferentes estações do ano (Cavalcanti 1974; Maimoni-Rodella \& Gottsberger 1980; Rodrigues \& Guerrero 1990).

As famílias melhor representadas foram Physaraceae e Stemonitaceae, às quais pertencem $60 \%$ das espécies, seguidas por Trichiaceae (20\%); as demais famílias estão representadas por uma a duas espécies cada (Tabs. 1-2).

Registrou-se predominância de espécies lignícolas, frutificando sobre troncos mortos de dicotiledôneas ( $45 \%$ ), com baixa incidência sobre folhedo (22\%) e uma terça parte ocorrendo em restos de estipe e folhas mortas de palmeiras (33\%). Ceratiomyxa fruticulosa e Hemitrichia calyculata foram as únicas espécies encontradas nos três grupos de substrato ; as duas espécies de Didymium assinaladas na área estudada foram coletadas unicamente em folhedo, cuja biomassa, constituída principalmente de folhas (ca. $80 \%$ ), corresponde em média a $407 \mathrm{~kg} / \mathrm{ha}$, com teor médio de umidade de $25 \%$ por ocasião das coletas.

Nas Tabs. 3-4 estão relacionadas as espécies ocorrentes no município do Crato, sua área de distribuição conhecida para o Nordeste do Brasil e respectivas fontes bibliográficas.

Dentre as espécies assinaladas, algumas são muito comuns, como Arcyria cinerea, Ceratiomyxa fruticulosa, Dictydium cancellatum, Fuligo septica e Perichaena depressa, de ampla distribuição, não somente na Região Nordeste, como também no Brasil e em

Tabela 1. Ceratiomyxales, Liceales e Trichiales ocorrentes na Chapada do Araripe (Crato, CE) e respectivos substratos de frutificação

\begin{tabular}{|c|c|c|c|}
\hline \multirow[t]{2}{*}{ TÁXONS } & \multicolumn{3}{|c|}{ SUBSTRATO } \\
\hline & Troncos mortos & Palmeiras mortas & Folhedo \\
\hline \multicolumn{4}{|l|}{ CERATIOMYXACEAE } \\
\hline Ceratiomyxa fruticulosa ( Mull.) Macbr. & + & + & + \\
\hline \multicolumn{4}{|l|}{ ENTERIDIACEAE } \\
\hline Dictydiaethalium plumbeum (Schum.) Rost. & + & - & - \\
\hline \multicolumn{4}{|l|}{ CRIBRARIACEAE } \\
\hline Cribraria tenella Schrd. & + & - & - \\
\hline Dictydium cancellatum ( Batsch) Macbr. & + & - & - \\
\hline \multicolumn{4}{|l|}{ TRICHIACEAE } \\
\hline Perichaena depressa Lib. & + & - & - \\
\hline Hemitrichia calyculata (Speg.) Farr & + & + & + \\
\hline H. serpula ( Scop.) Rost. & $\cdot$ & - & + \\
\hline Arcuria cinerea (Bull.) Pers. & + & - & - \\
\hline A. insignis Kalch. \& Cke. & + & - & - \\
\hline A. magna var. rosea $\mathrm{Rex}$ & - & + & - \\
\hline
\end{tabular}


Tabela 2. Physarales e Stemonitales ocorrentes na Chapada do Araripe ( Crato, CE ) e respectivos substratos de frutificação

\begin{tabular}{lcc}
\hline TÁXONS & SUBSTRATO & \\
Troncos mortos & Palmeiras mortas & Folhedo \\
\hline
\end{tabular}

PHYSARACEAE

Fuligo septica (L.) Wigg.

Fuligo sp.

Badhamia affinis Rost.

B. macrocarpa (Ces.) Rost.

Physarum compressum Alb. \& Schw.

P. mucelatum Rex

P. nutans Pers.

P. stellatum (Mass.) Martin

Physarum sp.

DIDYMIACEAE

Didymium difforme (Pers.) S.F.Gray

D. iridis (Ditmar) Fries

\section{STEMONITACEAE}

Comatricha irregularis Rex

C. longa Peck

C. typhoides (Bull.) Rost.

Stemonitis axifera (Bull.) Macbr.

S. flavogenita Jahn

S. herbatica Peck

S. pallida Wing.

S. splendens Rost.

Stemonitis sp.

Presente no substrato: +

$\begin{array}{cc}- & + \\ + & - \\ - & + \\ - & + \\ - & - \\ - & + \\ + & + \\ + & +\end{array}$
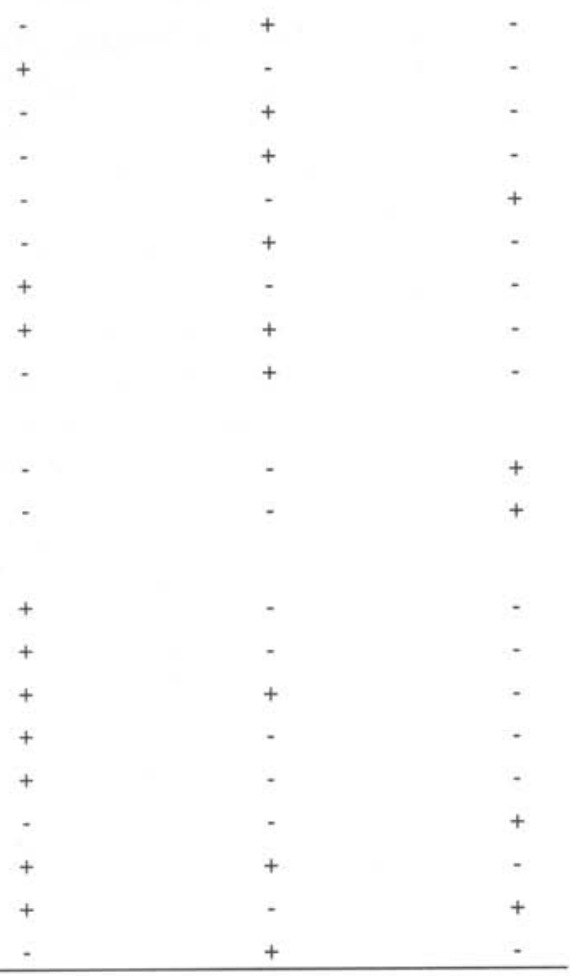

Não registrado no substrato: -

diferentes países do mundo. Outras, como Hemitrichia calyculata, apresentam também ampla distribuição no Brasil, porém são mais restritas às regiões tropicais.

O primeiro registro de ocorrência de Comatricha irregularis para o Brasil baseouse em amostra coletada em 1951 na Mata de Dois Irmãos, em Recife, PE (IPA 2987); identificada por A. C. Batista, foi considerada por Pôrto (1982) como duvidosa. O material coletado no Crato confirma que o Nordeste do Brasil está incluído na área de distribuição da referida espécie, que abrange a Ásia, Austrália, América do Norte, América Central e América do Sul.

Dentre as espécies listadas, apenas 11 já haviam sido referidas para o Ceará (Alves \& Cavalcanti 1996), as demais são novas referências para o Estado, ampliando o conhecimento sobre sua distribuição no Brasil (Tab. 3-4). Badhamia macrocarpa está sendo referida pela primeira vez para a região. O registro da família Enteridiaceae complementa as informações de Cavalcanti \& Brito Jr. (1990) sobre a distribuição deste táxon no Brasil, sendo Dictydiaethalium plumbeum espécie de ocorrência pouco conhecida no país. 
Tabela 3 - Ceratiomyxales, Liceales e Trichiales da Chapada do Araripe (Crato, CE): distribuição geográfica no Nordeste do Brasil.

\begin{tabular}{|c|c|c|c|c|c|c|c|c|c|c|}
\hline \multirow[t]{2}{*}{ TÁXONS } & \multicolumn{9}{|c|}{ Estados do Brasil } & \multirow[t]{2}{*}{ Referências } \\
\hline & BA & SE & $\mathrm{AL}$ & PE & PB & RN & $\mathrm{CE}$ & MA & PIP & \\
\hline Ceratiomyxa fruticulosa (Mull.) Macbr. & + & - & + & + & - & - & + & - & - & $1 ; 2 ; 5 ; 7$ \\
\hline Dicțdiaethalium plumbeum (Schum.) Rost. & - & - & - & + & - & - & + & - & - & 2 \\
\hline Cribraria tenella Schrd. & - & - & - & + & - & - & + & - & - & 2 \\
\hline Dictydium cancellatum (Batsch) Macbr. & + & - & - & + & + & - & + & - & - & $1 ; 2 ; 3 ; 7$ \\
\hline Perichaena depressa Lib. & + & - & - & + & + & - & + & - & - & $1 ; 2 ; 4$ \\
\hline Hemitrichia calyculata (Speg.) Farr & + & - & - & + & + & - & + & - & - & $1 ; 2 ; 4 ; 7$ \\
\hline H. serpula (Scop.) Rost. & + & - & - & + & + & - & + & - & - & $1 ; 2 ; 4 ; 7$ \\
\hline Arcyria cinerea (Bull.) Pers. & + & - & + & + & + & - & + & - & - & $1 ; 2 ; 4 ; 5$ \\
\hline A. insignis Kalch. \& Cke. & + & - & - & + & + & - & + & - & - & $2 ; 4 ; 6$ \\
\hline A. magna var. rosea Rex & - & . & - & - & - & - & + & - & - & \\
\hline
\end{tabular}

REFERÉNCIAS: 1-Torrend (1916): 2-Cavalcanti (1976): 3-Cavalcanti \& Araújo (1985): 4-Cavalcanti \& Marinho (1985): 5-Santos et al. (1986): 6-Góes Neto (1994): 7-Alves \& Cavalcanti (1996)

Tabela 4 - Physarales e Stemonitales da Chapada do Araripe (Crato. CE):distribuição geográfica no Nordeste do Brasil

\begin{tabular}{|c|c|c|c|c|c|c|c|c|c|c|}
\hline \multirow[t]{2}{*}{ TÁXONS } & \multicolumn{9}{|c|}{ Estados do Brasil } & \multirow[t]{2}{*}{ Referências } \\
\hline & $\mathrm{BA}$ & SE & $\mathrm{AL}$ & $\mathrm{PE}$ & $\mathrm{PB}$ & $\mathrm{RN}$ & $\mathrm{CE}$ & MA & PIP & \\
\hline Fuligo septica (L.) Wigg. & + & - & + & + & - & - & + & - & - & $2 ; 3 ; 7 ; 9$ \\
\hline Fuligo sp. & - & - & - & - & - & - & + & - & - & \\
\hline Badhamia affinis Rost. & - & - & - & + & - & - & + & - & - & $3 ; 9$ \\
\hline B. macrocarpa (Ces.) Rost. & - & - & - & - & - & - & + & - & - & \\
\hline Physarum compressum Alb. \& Schw. & + & - & - & + & - & - & + & - & - & $3 ; 8$ \\
\hline P. nucleatum Rex & - & - & - & + & - & $\cdot$ & + & - & - & $3 ; 9$ \\
\hline P. nutans Pers. & + & - & - & + & - & - & + & + & - & $1 ; 2 ; 3$ \\
\hline P. stellatum (Mass.) Martin & + & - & - & + & - & - & + & - & - & $2 ; 3 ; 9$ \\
\hline Phỵsarum sp. & - & - & - & $\cdot$ & - & - & + & - & - & 9 \\
\hline Didsmium difforme (Pers.) S.F. Gray & + & - & - & + & - & - & + & - & - & $3 ; 4$ \\
\hline D. iridis (Ditmar) Fries & + & - & - & + & - & - & + & + & - & $1 ; 2 ; 3$ \\
\hline Comatricha irregularis Rex & - & - & - & + & - & - & + & - & - & 5 \\
\hline C. Ionga Peck & + & - & - & + & - & - & + & - & - & $2 ; 3$ \\
\hline C. typhoides (Bull.) Rost. & - & - & - & + & - & - & + & - & - & $3 ; 9$ \\
\hline Stemonitis axifera (Bull.) Macbr. & + & - & - & + & - & - & + & - & - & $2 ; 3$ \\
\hline S. flavogenita Jahn & + & - & - & + & + & - & + & - & - & $3 ; 6$ \\
\hline S. herbatica Peck & - & - & - & + & + & - & + & - & - & $3 ; 6$ \\
\hline S. pallida Wing. & - & - & - & + & - & - & + & - & - & $3 ; 9$ \\
\hline S. splendens Rost. & - & - & + & + & - & - & + & - & - & $3 ; 7$ \\
\hline Stemonitis sp. & - & - & - & - & - & - & + & - & - & 9 \\
\hline
\end{tabular}


Uma das amostras do gênero Arcyria , encontrada no Sítio Fundão, foi identificada como A. magna var. rosea, descrita por Rex em 1893 ( Martin \& Alexopoulos 1969). O aspecto macroscópico e a coloração dos corpos frutíferos são muito semelhantes ao ilustrado por Yamamoto \& Nannenga-Bremekamp (1995), que a referem para o Japão; os referidos autores descrevem os corpos frutíferos como rosados, fusionados em pequenos grupos pelos longos pedicelos, de calículo longo-afunilado, margens levemente revolutas, com verrugas e papilas distribuídas em padrão subreticulado na face interna, caracteres também observados no material do Ceará. A. magna var. magna, embora pouco comum, é referida para os Estados de Alagoas, Paraíba, Pernambuco e São Paulo, constituindo este o primeiro registro da var. rosea para o Brasil.

Chave para identificação das espécies de Myxomycetes ocorrentes na Chapada do Araripe, município do Crato, CE.

1. Esporos localizados externamente à frutificação... Ceratiomyxa fruticulosa

1. Esporos internos à frutificação

2. Esporada pálida ou de colorido claro; esporos hialinos, amarelados ou amareloacastanhados sob luz transmitida

2. Esporada preta ou fortemente castanho-púrpura, castanha ou castanho-violácea sob luz transmitida

3. Capilício verdadeiro presente .................................................................... 4

3. Capilício verdadeiro ausente; pseudocapilício às vezes presente ............... 9

4. Filamentos do capilício com 2-6 bandas espirais bem definidas, lisas ou espinescentes 5

4. Filamentos do capilício lisos ou ornamentados, sem espirais 6

5. Esporângios pedicelados; capilício liso ou minutamente espinuloso; esporos delicadamente reticulados Hemitrichia calyculata

5. Plasmodiocarpos; capilício espinuloso; esporos nitidamente reticulados ..... Hemitrichia serpula

6. Capilício formado por filamentos livres, simples ou ramificados, não formando rede; esporângios sésseis, depressos Perichaena depressa

6. Capilício formado por filamentos longos, anastomosados, formando rede; esporângios pedicelados 7

7. Esporângios brancos, acinzentados ou ocráceos; capilício densamente espinhoso no ápice da frutificação, mais largo e liso na base

Arcyria cinerea

7. Esporângios vermelhos, salmão, rosados a amarelados 8

8. Capilício espinescente, firmemente preso ao calículo raso; pedicelos livres, esporângios em pequenos tufos Arcyria insignis

8. Capilício com meio anéis, desprendendo-se na maturidade do calículo profundo; esporângios unidos pelos pedicelos 
9. Grânulos dictidinos ausentes; pseudoetálios; porções do perídio persistindo sem formar rede

Dictydiaethalium plumbeum

9. Grânulos dictidinos presentes; esporangiados; porções do perídio persistindo como rede pré-formada

10. Filamentos da rede peridial curtos, encontrando-se em nódulos regulares ... Cribraria tenella

10. Filamentos da rede peridial longitudinais, longos, subparalelos, conectados por filamentos transversais delicados

Dictydium cancellatum

11. Perídio totalmente evanescente, mas deixando rede superficial unida ao capilício

11. Perídio evanescente ou persistente na base, não deixando rede superficial ..

12. Esporângios profundamente fuscos ou pretos Stemonitis splendens

12. Esporângios castanhos a vináceos ou pálidos 13

13. Esporos quase lisos, atingindo 5-7 $\mu \mathrm{m}$ diâm.; esporângios com 7-15mm alt. Stemonitis axifera

13. Esporos distintamente espinulosos, usualmente maiores que $7 \mu \mathrm{m}$ diâm.; esporângios com 3-8mm alt.

14. Esporângio castanho-claro; pedicelo raramente atingindo $1 / 3$ da altura total; rede superficial persistente no ápice Stemonitis herbatica

14. Esporângio castanho-escuro ou pálido; pedicelo usualmente 1/3 ou mais da altura total; rede superficial fugaz no ápice

15. Corpo frutífero castanho; columela originando muitos ramos grosseiros, tendendo a se expandir no ápice Stemonitis flavogenita

15. Corpo frutífero pardacento ou pálido; columela dispersa bem abaixo do ápice, filamentos do capilício delicados Stemonitis pallida

16. Cálcio presente, em grânulos ou cristais, distribuído em diferentes partes do corpo frutífero

16. Cálcio ausente nos corpos frutíferos

17. Capilício intrincado, com muitas anastomoses, as quais formam rede densa, com poucas pontas livres ou estas ausentes; perídio persistente na base

Comatricha typhoides

17. Capilício aberto, com poucas ou nenhuma anastomose, eventualmente formando rede muito aberta

18. Esporângio fusco-escuro a preto, com 10-50 mm alt.; capilício com poucas anastomoses; esporos conspicuamente verrucoso-reticulados

Comatricha longa

18. Esporângio castanho-escuro a preto, $2-8 \mathrm{~mm}$ alt.; capilício aberto, com poucas anastomoses, com pontas muito longas mas raramente anastomosadas; esporos verrucosos 
19. Cálcio presente sob a forma de cristais, distribuído no pedicelo, perídio e/ou columela, caracteristicamente ausente no capilício ..................................... 26

19. Cálcio presente na forma de grânulos, distribuído no pedicelo, perídio e/ou columela, caracteristicamente presente no capilício

20. Etálio cinzento, amarelado ou alaranjado. Fuligo septica

20. Esporângio cinzento ou branco- acinzentado 21

21. Capilício totalmente formado por túbulos calcários ou com uns poucos filamentos não calcários interligantes

21. Capilício formado por filamentos hialinos que conectam nódulos calcários

22. Esporos irregularmente verrucosos; pedicelo, quando presente, amarelado ou acastanhado, exceto na base, onde se apresenta mais escuro

Badhamia macrocarpa

22. Esporos densamente espinulosos; pedicelo, quando presente, preto

Badhamia affinis

23. Pseudocolumela presente, globosa ou irregular, calcária 24

23. Pseudocolumela ausente 25

24. Pedicelo calcário; esporos 8,0-10,0 $\mu$ m diâm Physarum stellatum

24. Pedicelo não calcário; esporos 6,5-7,5 $\mu \mathrm{m}$ diâm Physarum nucleatum

25. Esporângios depresso-esféricos a lenticulares, umbilicados; esporos rugosos Physarum nutans

25. Esporângios compresso-globosos, compresso-reniformes, algumas vezes lobados; esporos minutamente espinulosos

Physarum compressum

26. Perídio coberto por crosta firme de cristais de cálcio; columela presente; plasmodiocárpicos Didymium difforme

26. Perídio coberto por crosta pulverulenta de cristais de cálcio; columela ausente; esporângios pedicelados

Didymium iridis

\section{Agradecimentos}

Os autores agradecem aos professores Francisco A. B. da Cunha e Terezinha Gonçalves Batista, da Universidade Regional do Cariri (URCA), pelo apoio logístico para as excursões e a atenção dispensada durante a estada na cidade do Crato.

\section{Referências bibliográficas}

Alexopoulos, C. J. 1970. Rain Forest Myxomycetes. Pp. 21-23. In: H. T. Odum (ed.). A Tropical Rain Forest, U.S. Atomic Energy Comission.

Alves. M. H. \& Cavalcanti. L. de H. 1996. Myxomycetes em palmeiras (Arecaceae). Acta Botanica Brasilica 10(1): 1-7

Cavalcanti, L. de H. 1974. Mixomicetos corticícolas do cerrado de Emas (Pirassununga - São Paulo).

Dissertação de Mestrado. Universidade de São Paulo, São Paulo. 
Cavalcanti, L. de H. 1976. Mixomicetos novos para Pernambuco. II. Universidade Federal de Pernambuco, Ciências Biológicas, Recife, Série Botânica, 4(4): 1-19.

Cavalcanti, L. de H. 1996. Myxomycetes. Cap. 3. Pp. 37-45. In E. V. S. B. Sampaio; S. J. Mayo \& M. R. V. Barbosa (Coord.), Pesquisa Botânica Nordestina: progresso e perspectivas. Sociedade Botânica do Brasil, Recife.

Cavalcanti, L. de H. \& Araújo. V. C. F. 1985. Myxomycetes da Paraiba II- Liceales. Pp. 193 -198. In: Anais da VIII Reunião Nordestina de Botânica. Sociedade Botânica do Brasil. Seccional Pernambuco, Recife.

Cavalcanti, L. de H. \& Brito Júnior, S. C. 1990. Enteridiaceae do Brasil. Biologica Brasilica 2(2): 241- 260.

Cavalcanti, L. de H. \& Marinho, M. das G. V. 1985. Myxomycetes da Paraíba I- Trichiales. Pp. 185 - I9I. In: Anais da VIII Reunião Nordestina de Botânica. Sociedade Botânica do Brasil, Seccional Pernambuco, Recife.

Cavalcanti, L. de H. \& Oliveira. I. C. 1985. Myxomycetes da Paraiha IV- Stemonitales. Pp. 297 - 214. In: Anais da VIII Reunião Nordestina de Botânica. Sociedade Botânica do Brasil, Seccional Pernambuco. Recife.

Farr. M. L. 1960. The Myxomycetes of the IMUR Herbarium. with special reference to Brazilian species. Publicações do Instituto de Micologia 184: 1-54.

Farr, M. L. 1976. Myxomycetes. Flora Neotropica . Monograph 16. New York Botanical Garden, New York.

FIBGE-Fundação Instituto Brasileiro de Geografia e Estatística. 1977. Diretoria Técnica. Geografia do Brasil. Região Nordeste. vol. 2. SERGRAF, IBGE. Rio de Janciro.

Góes-Neto, A. 1994. Diagnóstico da biodiversidade de macromicetos do Estado da Bahia: revisão histórica e quadro atual. Monografia de Bacharelado. Universidade Federal da Bahia, Salvador.

Gottsberger, G. 1968. Myxomyceten aus Bahia und Goiás. Nova Hedwigia 15: 361-368.

Lister. A. 1925. A monograph of the Mycetozoa. British Museum. Natural History, London.

Maimoni - Rodella, R. de C. S. \& Gottsberger. G. 1980. Myxomycetes from the forest and the cerrado vegetation in Botucatu. Brazil: a comparative ecological study. Nova Hedwigia 34: 207-248.

Martin, G. W. \& Alexopoulos. C. J. 1969. The Myxomycetes. University of Iowa Press, Iowa.

Martin. G. W.; Alexopoulos. C. J. \& Farr, M. L. 1983. The Genera of Myxomycetes. University of Iowa Press, Iowa.

Pôrto. K.C. 1982. Myxomycetes da Mata de Dois Irmãos ( Recife - Pernambuco). Dissertação de Mestrado. Universidade Federal Rural de Pernambuco. Recile.

Rodrigues, C. L. M. \& Guerrero. R.T. 1990. Myxomycetes do Morro Santana, Porto Alegre, Rio Grande do Sul. Boletim do Instituto de Biociências 45: 1-102.

Santos. E. J.: Cavalcanti. L. de H. \& Albuquerque, W. C. 1986. Myxomycetes de Alagoas. Pp. 499 -504. In: Anais do XXXIII Congresso Nacional de Botânica. Universidade Federal de Ouro Preto, Ouro Preto.

SUDEC. 1972. A microrregião plana do Cariri. Fortaleza: Convênio SUDENE/ Estado do Ceará/ SERFHAU.

Torrend. C. 1915. Les Myxomycètes du Brasil, connus jusquici. Broteria, Série Botânica 13: 72 - 88.

Torrend. C. 1916. Os mixomicetas dos arredores da Bahia. Pp. 484-492. In: Anais do V Congresso Brasileiro de Geografia. Sociedade Brasileira de Geografia, Salvador.

Yamamoto,Y. \& Nannenga - Bremekamp. N. E. 1995. Additions to the Myxomycetes of Japan V. Proceedings of the Koninklijke Nederlandese Akademie van Wetenschappen 98(3): 317-326. 